\title{
LONG-TERM CONSEQUENCES OF PRIMARY PERCUTANEOUS CORONARY INTERVENTION IN PATIENTS WITH ST-ELEVATION MYOCARDIAL INFARCTION
}

DOI: $10.36740 /$ WLek202004107

\author{
Nataliya V. Izhytska, Dmytro I. Besh, Olesya M. Besh, Adrian Y. Fil \\ DANYLO HALYTSKY LVIV NATIONAL MEDICAL UNIVERSITY, LVIV, UKRAINE
}

\begin{abstract}
The aim: To investigate long-term effects of primary percutaneous coronary interventions ( $\mathrm{pPCl}$ ) in patients with STEMI basing on the prevalence of clinically relevant endpoints. Materials and methods: Totally 200 patients with STEMI hospitalized within a period of "therapeutic window" for revascularization were included into the study. 100 patients who additionally to $\mathrm{pPCl}$ underwent manual thromboaspiration entered the main group. The comparison group consisted of 100 patients who underwent standard pPCl. Results: Six months after the pPCI, the incidence of either major adverse cardiac events and the combined endpoint did not differ in the main and comparative groups (11.8\% vs. $12.5 \%, p=0.888$ and $18.2 \%$ vs. $20.8 \%, p=0.658$, respectively). There were no significant differences in these endpoints taken separately. In twelve months after procedure, also there were no significant differences between the groups. However, a tendency toward lower incidence of chest pain was observed in the main group $(p=0.08)$ during this period that was lost in 24 months after PPCI. None of these techniques demonstrated significant advantages during the whole duration of the follow-up period.

Conclusions: The addition of manual thromboaspiration to the standard PPCI in patients with STEMI and severe thrombosis of the culprit artery did not significantly influence the prognosis.
\end{abstract}

KEY WORDS: manual thromboaspiration, prognosis, acute ST-elevation myocardial infarction, primary percutaneous coronary intervention

Wiad Lek. 2020;73(4):662-667

\section{INTRODUCTION}

Cardiovascular diseases cause approximately $40 \%$ of all mortality cases in the population of most developed European countries. Ischemic heart disease (IHD) occupies a leading place in the structure of this pathology, and acute myocardial infarction is one of the main causes of death and patients' disability [18]. The main cause is coronary artery thrombosis, which is thought to be caused by rupture of atherosclerotic plaque. In present-day clinical practice, different varieties of acute myocardial infarction are distinguished. Their clinical, laboratory and functional characteristics are described in medical literature, which significantly facilitates an algorithm of diagnostic search. Nowadays it has been proven that the highest indices of mortality among patients with IHD are due to ST-elevation myocardial infarction (STEMI) [2,3].

In the recent years, one of the basic elements of STEMI treatment has been conduction of primary percutaneous coronary intervention (pPCI). However, there are publications, which show that adequate reperfusion cannot always be achieved even with its use. One of the main causes of this phenomenon is development of distal embolism, which, in its turn, results in microvascular obstruction. The latter causes increase in the frequency of early post-infarction complications, negative remodeling of the left ventricle, late recurrent hospitalizations, caused by cardiac insufficiency and mortality $[4,5]$.
To prevent distal embolism in pPCI, a series of measures were suggested, namely, mechanic thrombus aspiration, manual thrombus aspiration and embolic protection. In the recent years, meta-analysis of investigations, which compared the efficacy of the aforementioned methods, has been performed. It has been revealed that only addition of manual thrombus aspiration has certain advantages over a standard intervention technique. Two other methods proved ineffective, and in some cases even harmful [6]. The obtained results stimulated conduction of a series of investigations, which studied the efficacy of the aforementioned treatment strategy in patients with STEMI and showed ambiguous results.

Such situation dictates the need in further study of efficacy of various methods of pPCI conduction, in particular, based on analysis of follow-up observations.

\section{THE AIM}

To investigate long-term effects of primary percutaneous coronary interventions (pPCI) in patients with STEMI basing on the prevalence of clinically relevant endpoints.

\section{MATERIALS AND METHODS}

The investigation included 200 patients with STEMI, hospitalized within "therapeutic window" for revascular- 
Table I. Baseline characteristics of patients included in the study

\begin{tabular}{|c|c|c|c|c|c|}
\hline & \multicolumn{2}{|c|}{ Main group } & \multicolumn{2}{|c|}{ Control group } & \multirow{2}{*}{$\mathbf{p}$} \\
\hline & Number & $\%$ & Number & $\%$ & \\
\hline Male & 82 & 82 & 78 & 78 & 0,480 \\
\hline Diabetes mellitus & 23 & 23 & 13 & 13 & 0,066 \\
\hline Hypertension & 58 & 58 & 67 & 67 & 0,189 \\
\hline Obesity & 41 & 41 & 42 & 42 & 0,886 \\
\hline Dyslipidemia & 33 & 33 & 27 & 27 & 0,284 \\
\hline Smoking & 57 & 57 & 62 & 62 & 0,471 \\
\hline Contact with xenobiotic & 52 & 52 & 45 & 45 & 0,322 \\
\hline Cardiogenic shock & 12 & 12 & 7 & 7 & 0,228 \\
\hline Multivessel coronary diseases & 40 & 40 & 45 & 45 & 0,474 \\
\hline TIMI 0 flow before the intervention & 82 & 82 & 72 & 72 & 0,093 \\
\hline MBG 0 before the intervention & 88 & 88 & 81 & 81 & 0,287 \\
\hline Infarctrelated left coronary artery & 41 & 41 & 44 & 44 & 0,668 \\
\hline
\end{tabular}

ization. Occlusion of infarction-related coronary artery or its massive thrombosis was revealed on angiography in all participants. The main group included 100 patients, who had undergone manual thrombus aspiration in addition to standard pPCI. A control group included 100 patients, who had undergone intervention by standard technique. Efficacy of revascularization was assessed by the incidence of such indices: major adverse cardiac events (MACE) (death, myocardial infarction, recurrent revascularizations), combined endpoints (MACE or revascularization), as well as each element individually.

Blood flow by TIMI scale was studied according to standard technique: TIMI 0 - absent blood flow more distally than occlusion site; TIMI 1 - insignificant blood flow more distally from occlusion site, but distal regions of the artery are not filled; TIMI 2 - slow antegrade blood flow with complete filling of distal segments of the coronary artery; TIMI 3 - normal, complete filling of a distal part of the artery [7]. Myocardial blush was determined according to criteria elaborated by Van't Hof and co-authors: MBG 0 - absence of myocardial contrast opacification; MBG 1 - minimal myocardial contrast opacification; MBG 2 - moderate myocardial contrast opacification, however, lower than in angiography of contralateral or ipsilateral coronary artery unrelated to infarction; MBG 3 - normal myocardial contrast opacification, the same as in angiography of contralateral or ipsilateral coronary artery unrelated to infarction [8].

An important component of any treatment method efficacy is following by patients doctor's recommendations. We studied patients' adherence to treatment by means of a purposefully elaborated validated questionnaire (8-items Morisky Medication Adherence Scale) [9]. The variation used was elaborated in 2008 year and includes 8 questions, which promotes significant reduction of risk for patients' voluntary exaggeration of their adherence to treatment. It enables 3-degree assessment of adherence to treatment, explaining it as high, average and low [10]. Questioning of patients was performed via phone calls or during visits to a clinic. All investigated indices were studied in 6,12 and 24 months after hospitalization.

Statistical analysis of the obtained results was conducted on a computer using program $\mathrm{R}$. The obtained results were presented as:

- mean values and their standard deviations $(\mathrm{M} \pm \mathrm{SD})-$ in case of Gaussian distribution,

- medians, $25^{\text {th }}$ and $75^{\text {th }}$ percentiles: Me [25\%; 75\%] - in case of non-Gaussian distribution,

- in analysis of relative values, creation of $2 \times 2$ tables and calculation of percentage with its estimated faulty proportion was performed $\left(\mathrm{P} \pm \mathrm{m}_{\mathrm{p}}\right)$.

On assessment of difference reliability of obtained results in investigated groups, the following means were used:

- unpaired t-criterion - for two groups with Gaussian distribution;

- Mann-Whitney U test - for two groups with non-Gaussian distribution;

- criterion $\chi^{2}$ (chi-square) - in comparison of percentages.

The difference between groups was considered reliable at values $\mathrm{p}<0.05$.

\section{RESULTS}

Patients of both groups reliably did not differ by primary characteristics. The age of investigated patients in the main group constituted $58.15 \pm 11.34$ versus $57.81 \pm 10.26$ years in control group, $(\mathrm{p}=0.824)$; differences in gender characteristics of both groups were not observed $(\mathrm{p}=0.480)$. Analysis of risk factors of IHD did not reveal statistically reliable difference between groups in the incidence of diabetes mellitus ( $\mathrm{p}=0.066)$, arterial hypertension $(\mathrm{p}=0.189)$, obesity $(\mathrm{p}=0.886)$, dyslipidemia $(\mathrm{p}=0.284)$, smoking $(\mathrm{p}=0.471)$ and professional exposure to xenobiotics $(\mathrm{p}=0.322)$. Assessing the severity of patients' conditions on admission to hospital, the incidence of cardiogenic shock development was studied. It was diagnosed somewhat more frequently in the 
main group, however, the difference was not statistically reliable ( $12 \%$ versus $7 \%, \mathrm{p}=0.228)$. Analysis of angiography demonstrated similar incidence of thrombosis of left coronary artery $(\mathrm{p}=0.668)$ and multivessel coronary diseases $(\mathrm{p}=0.474)$ in both groups. No reliable differences were revealed between groups $(p=0.093)$ in the incidence of TIMI 0 blood flow in the infarct related artery before pPCI. The incidence of blush degree MBG 0 detection before percutaneous revascularization in the region of blood supply to infarction-related coronary artery also did not differ reliably ( $\mathrm{p}=0.668)$ (Table I).

In 6 months after hospitalization, we managed to receive information about prognosis of 93 patients of the main group and 96 - in control group. Further analysis of long-term consequences is presented for these patients. The incidence of occurrence of MACE did not differ significantly between groups and constituted $11(11.8 \%)$ in the main group, and in control group - $12(12.5 \%)(\mathrm{p}=$ 0.888 ). Besides, no considerable changes were revealed between groups in relation to the incidence of combined endpoint registry (17-18.2\% versus $20-20.8 \%$; $p=0.658$ ). Analyzing each index individually, attention was primarily paid to the study of incidence of mortality cases registries. Throughout 6-month monitoring, 6 (6.5\%) patients in the main group and $3(3.1 \%)$ in control group died $(p=0.283)$. Incidence of other events was determined for alive patients, who could be reached. Throughout six months after hospitalization, myocardial infarction was diagnosed in one patient from each group (1.2\% versus $1.1 \% ; \mathrm{p}=0.962)$. No significant difference was observed in the incidence of recurrent revascularizations (coronary artery bypass grafting or recurrent percutaneous coronary intervention) in patients of the main group and control group (5.6\% versus $10.1 \%$ respectively; $p=0.282$ ). Patients of the main group noted recurrence of angina somewhat rarer at the first stage of monitoring $(10-11.5 \%$ and $17-18.3 \%$, respectively), though the difference was not statistically reliable $(\mathrm{p}=0.203)$.

Results of assessment of adherence to treatment, obtained from questionnaire enable to state that in patients of both groups it was at quite high level. In particular, it was high in $77(88.5 \%)$ patients of the main group and $82(88.2 \%)$ individuals of control group, which did not constitute reliable difference ( $\mathrm{p}=0.944)$ (fig. 4$)$.

In 12 months, we managed to obtain information about prognosis of 92 patients in the main group and 96 - in control group. Among them, the incidence of MACE did not significantly differ among groups and constituted $13(14 \%)$ in the main group and $17(17.7 \%)$ - in control group ( $\mathrm{p}=0.48)$. No significant differences were revealed in relation to the incidence of combined endpoint (25-27.2 $\%$ versus $37-38.5 \%$; $p=0.089$ ) (fig. 2 ).

Throughout 12 months of monitoring, 8 (8.6\%) patients of the main group and $6(6.25 \%)$ in control group died $(\mathrm{p}=0.537)$. The number of patients in both groups, who developed acute myocardial infarction, was the same and constituted $2(2.3 \%)$ patients in each group $(\mathrm{p}=0.982)$. The difference in the incidence of recurrent revascularizations remained in favor of patients of the main group, however, its indices did not reach statistical significance (5-5.8\% versus $9-10.1 \% ; \mathrm{p}=0.285)$. Besides, there was tendency in patients of the main group towards less probability of angina recurrence (14-16.1\% versus $24-27.0 \%$, respectively), though it was unreliable ( $\mathrm{p}=0.08$ ) (fig. 2).

In the period of 6 to 12 months of monitoring, number of patients with low adherence to treatment increased twice. Moreover, such situation equally concerned each group. High indices of adherence to treatment were detected in $66(75.9 \%)$ patients of the main group and $69(77.55 \%)$ patients of the control group ( $\mathrm{p}=0.794$ ) (fig.4).

In 24 months, we managed to obtain information on prognosis of 92 patients in the main group and 94 - in control group. Among them the incidence of occurrence of MACE did not differ significantly between groups and constituted $17(18.5 \%)$ in the main group and $20(21.3 \%)$ in the control one ( $p=0.633)$. No considerable differences were observed in relation to the incidence of combined endpoint (34-37.0\% versus $40-42.6 \%$, respectively; $p=0.436$ ) (fig.3).

Throughout 2-year monitoring, $9(9.8 \%)$ patients of the main group and $6(6.4 \%)$ - in control group died $(\mathrm{p}=0.395)$. No considerable difference was revealed in the incidence of occurrence of acute myocardial infarction (3-3.6\% versus $6-6.8 \%, \mathrm{p}=0.339)$. Recurrent revascularizations rarely occurred in both groups (8-9.5\% versus $10-11.4 \%$, respectively, $\mathrm{p}=0.694)$. Unreliable tendency towards lower incidence of angina recurrence in patients of the main group compared with the control group, which was noted in the first year of monitoring, was lost in the second year. Among patients of the main group this complaint was noted by $23(27.4 \%)$ patients of the main group and $27(30.7 \%)$ patients of the control group ( $\mathrm{p}=0.634)(\mathrm{p}=0.634)$ (fig. 3 ).

Throughout the period from 12 to 24 months of monitoring, minimal changes occurred in relation to adherence to treatment in both groups. High result by this index were observed in $62(73.8 \%)$ patients of the main group and 67 (76.1\%) patients of the control group ( $\mathrm{p}=0.725)$.

\section{DISCUSSION}

Aforementioned results of investigation did not demonstrate reliable benefit of manual thrombus aspiration in patients with STEMI and occlusion or massive thrombosis of infarct-related coronary artery. However, it cannot be stated that such result was unexpectable. Having analyzed evolution of investigations, which studied the efficacy of manual thrombus aspiration, we can see interesting peculiarities. In earlier investigations, benefits of such intervention were reported. An example is represented by single center study - The thrombus aspiration during percutaneous coronary intervention in acute myocardial infarction study (TAPAS), results of which were reported in 2008 year. They differed significantly from our results. TAPAS investigation demonstrated considerably lower indices of all-cause mortality, mortality due to cardiovascular pathology and incidence of occurrence of combined endpoint death + nonfatal myocardial infarction in patients who had undergone manual thrombus aspiration compared 


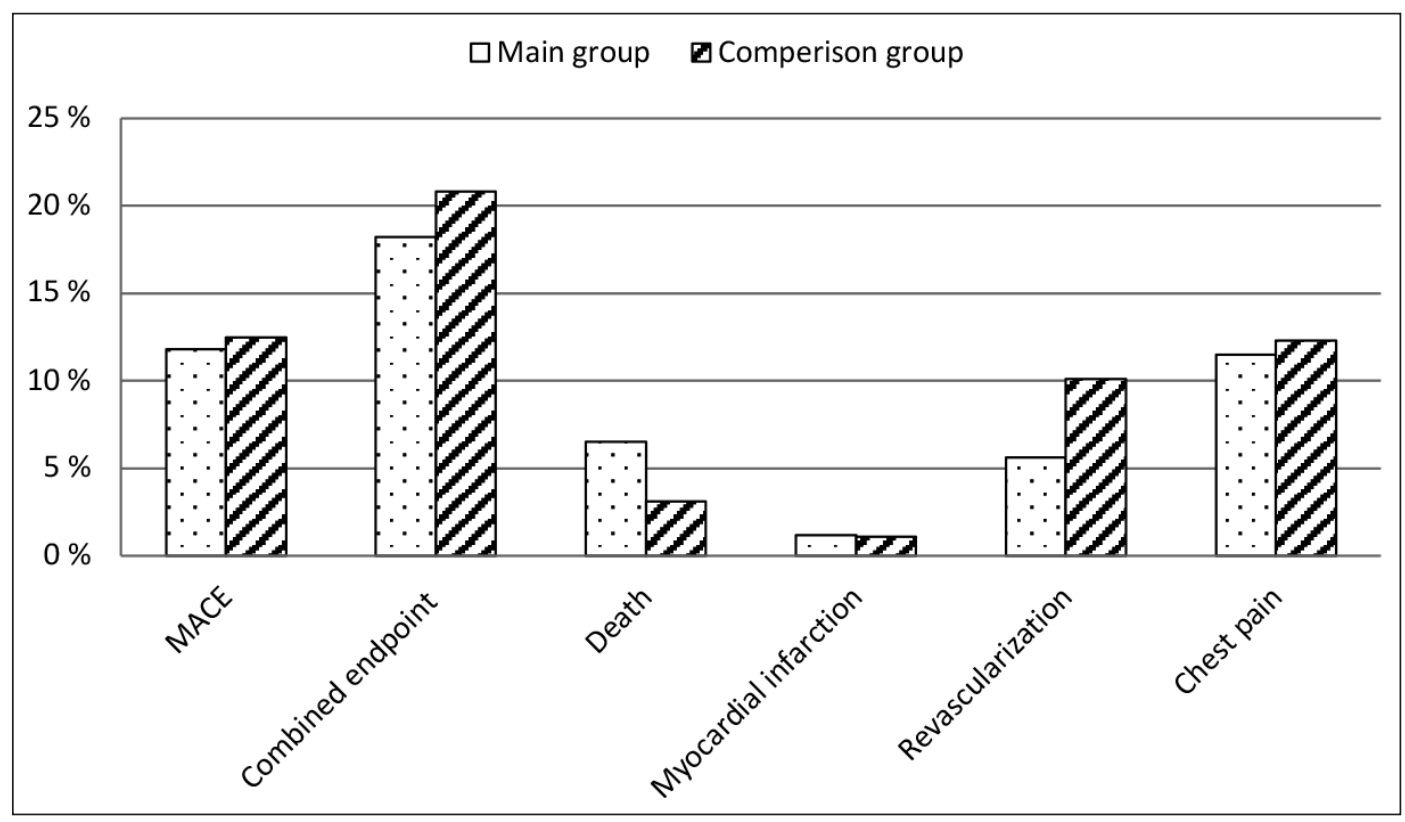

Fig.1. Incidence of development of investigated endpoints throughout 6-month monitoring

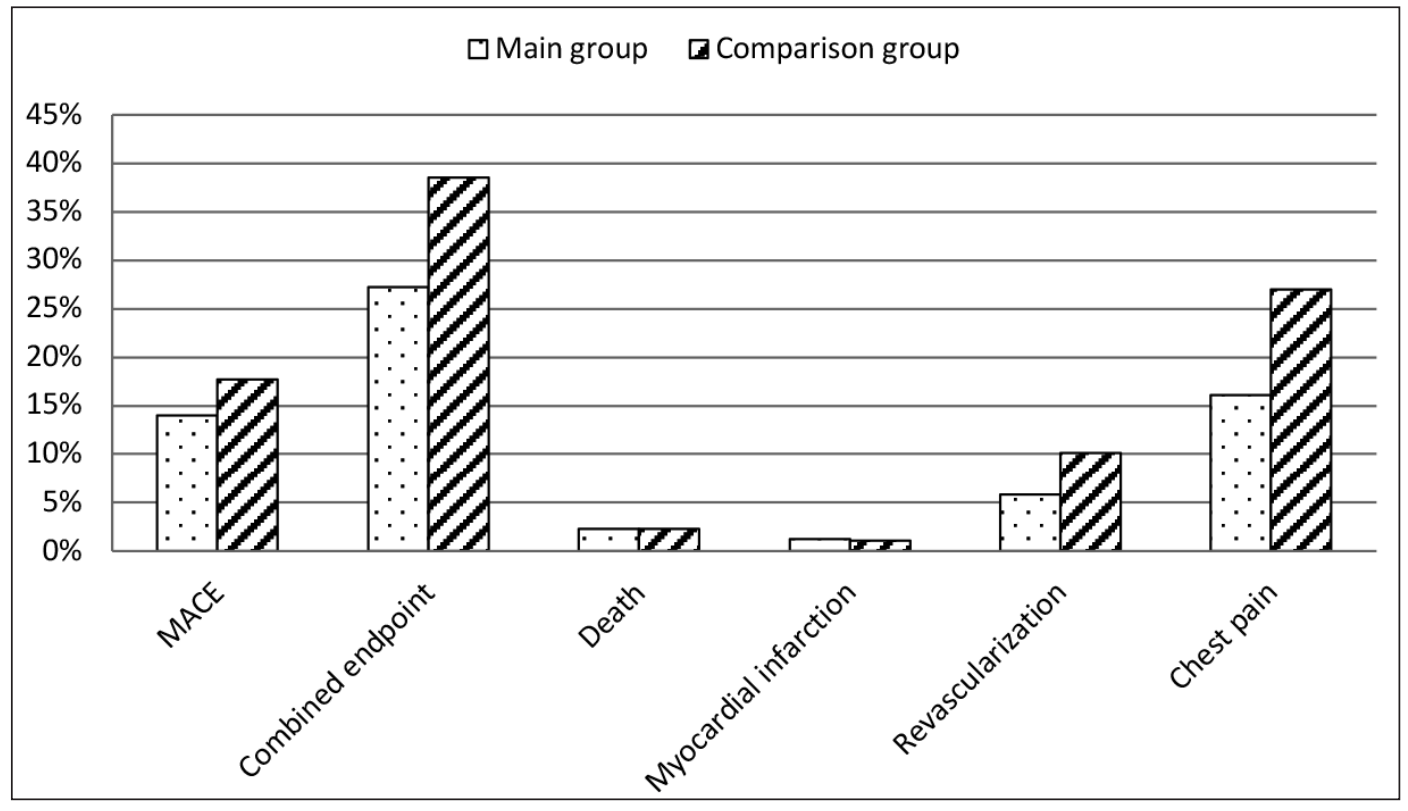

Fig.2. Incidence of development of investigated endpoints throughout 12 months of monitoring

with those who had undergone standard percutaneous revascularization [11]. It resulted in inclusion of the technique into guidelines of European Society of Cardiology for the management of patients with STEMI dated 2012 year, as well as recommendations of American Heart Association and American College of Cardiology with high class and evidence level $[12,13]$. However, there were series of remarks as to design of TAPAS investigation. Thus, it provoked conduction of multicenter study - Thrombus aspiration in ST-elevation myocardial infarction in Scandinavia (TASTE). Its results were reported in 2014. They did not reveal advantages of investigated intervention both in relation to development of myocardial infarction or thrombosis of a stent, as well as the risk of development of combined endpoint registry. Moreover, conduction of manual thrombus aspiration did not show efficacy in different subgroups of patients depending on age, gender, duration of angina syndrome, infarction-dependent coronary artery, duration of ischemia and concomitant pharmaceutical treatment [14]. Such result provoked reduction of evidence class for this type of intervention from IIa to IIb in guidelines of European Cardiology Society on revascularization in 2014 year [3]. More doubts about expediency of routine administration of manual thrombus aspiration appeared after revelation of investigation results in 2015 - The trial of routine aspiration thrombectomy with PCI versus PCI alone in patients with STEMI (TOTAL). It did not report any advantages of this intervention method over standard percutaneous coronary intervention either in general population or among certain subgroups of patients [15]. This resulted in denial of routine administration of manual thrombus aspiration in 


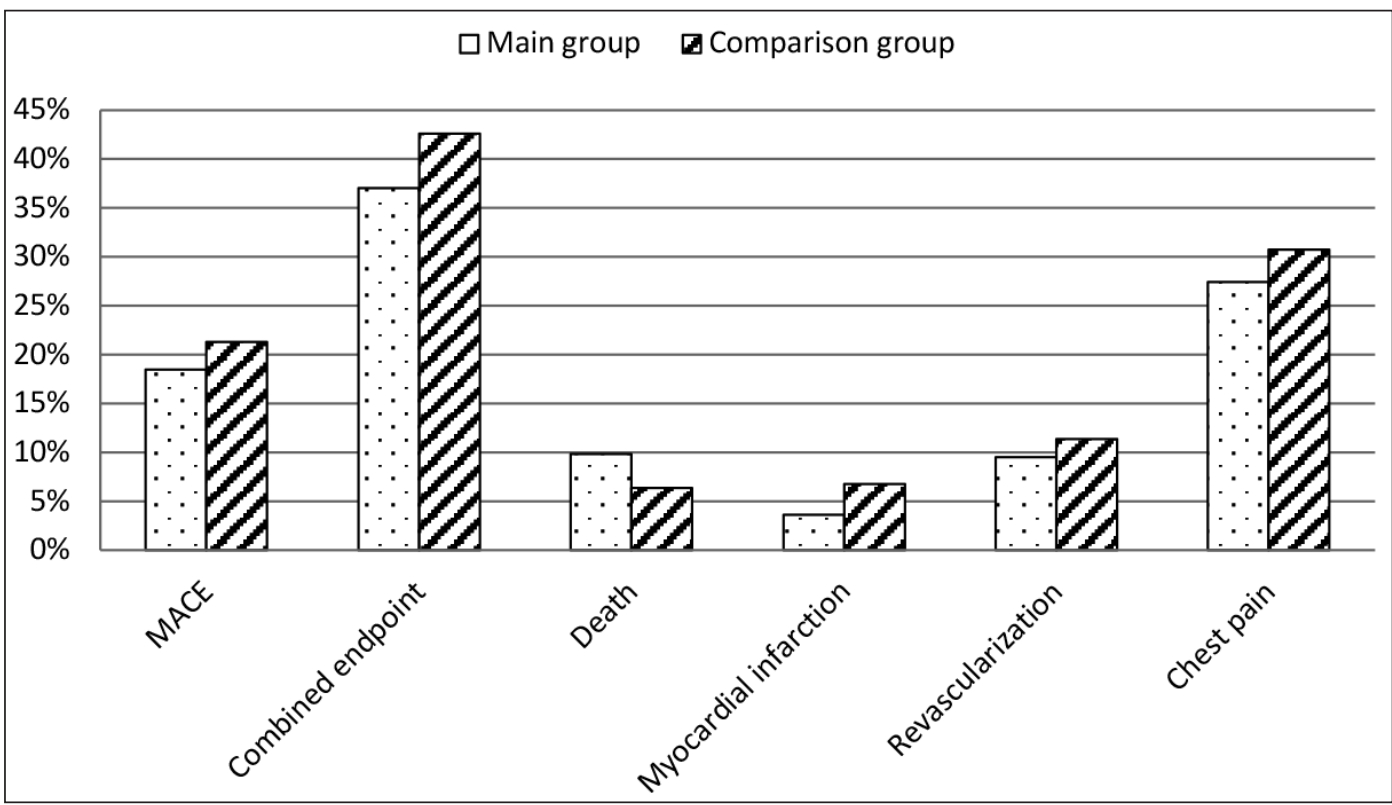

Fig 3. Incidence of development of investigated endpoints throughout 24 months of monitoring

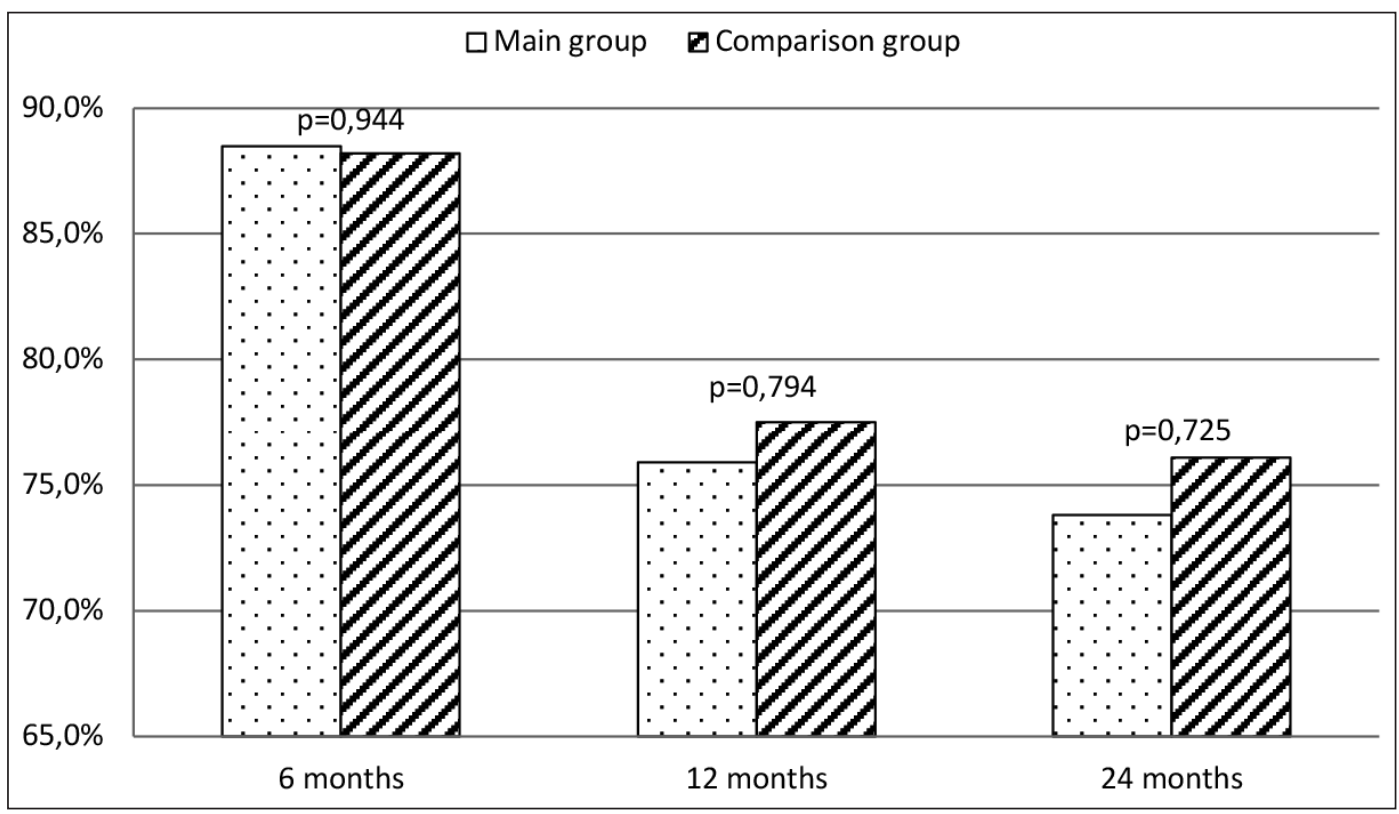

Fig. 4. Dynamics of patients' fraction with high adherence to treatment throughout investigation.

patients with STEMI both in topical guidelines of American Heart Association and American College of Cardiology for the management of patients with STEMI in 2017. However, both documents admitted possibility of using this technique in patients with additional indices $[1,16]$.

Results of two aforementioned investigations, as well as some others, conducted approximately at the same time, completely coincide with our data $[14,15,17]$. A significant difference implies that our investigation included not all patients with STEMI (routine administration), but only those with present occlusion of infarct-related coronary artery, or its marked thrombosis. However, even they did not reveal benefit from administration of additional manual thrombus aspiration. In our opinion, it can be explained by active use of new, powerful anti-platelet medicines as ticagrelor and prasugrel. Differences in results obtained in early investigations compared with modern ones can probably be explained by administration of such medicines.

An important element of effective treatment of patients with STEMI is patients' adherence to treatment. Our results showed rather high levels of adherence to treatment in patients of both groups. Taking into consideration significant influence of investigated intervention on patients' clinical condition, absence of difference in this index between groups is expectable. High indices of adherence to treatment among participants of our investigation can be explained by constant contact with an attending doctor and thorough dynamic monitoring of patients' conditions. Such approach, according to literature data, has significant influence on patients' adherence to treatment $[18,19,20]$. 


\section{CONCLUSIONS}

Administration of manual thrombus aspiration in patients with STEMI after pPCI did not have significant influence on prognosis, even in the presence of marked thrombosis of the coronary arteries.

\section{REFERENCES}

1. Ibanez B., James S., Agewall S. et al. 2017 ESC Guidelines for the management of acute myocardial infarction in patients presenting with ST-segment elevation. European Heart Journal. 2017;39(2):119-177.

2. Mozaffarian D., Benjamin E., Go A. et al. (2016). Heart Disease and Stroke Statistics - 2016 Update. Circulation, 2016;133(4):e38-360.

3. Windecker S., Kolh P., Alfonso F. et al. 2014 ESC/EACTS Guidelines on myocardial revascularization: The Task Force on Myocardial Revascularization of the European Society of Cardiology (ESC) and the European Association for Cardio-Thoracic Surgery (EACTS) Developed with the special contribution of the European Association of Percutaneous Cardiovascular Interventions (EAPCI). European Heart Journal. 2014;35(37):2541-619.

4. NiccoliG.,BurzottaF.,Galiuto L.etal.Myocardial No-Reflowin Humans.Journal of the American College of Cardiology. 2009;54(4):281-292.

5. Crea F. Coronary Microvascular Obstruction - A Puzzle with Many Pieces. New England Journal of Medicine. 2015;372(15):1464-1465.

6. Bavry A., Kumbhani D., Bhatt D. Role of adjunctive thrombectomy and embolic protection devices in acute myocardial infarction: a comprehensive meta-analysis of randomized trials. European Heart Journal. 2008;29(24):2989-3001.

7. The Thrombolysis in Myocardial Infarction (TIMI) Trial. New England Journal of Medicine. 1985;312(14):932-936.

8. van't Hof A., Liem A., Suryapranata H. et al. Angiographic Assessment of Myocardial Reperfusion in Patients Treated With Primary Angioplasty for Acute Myocardial Infarction. Circulation. 1998;97(23):2302-2306.

9. Lahdenperä T., Kyngäs H. Compliance and its evaluation in patients with hypertension. Journal of Clinical Nursing, 2000;9(6):826-833.

10. Morisky D., Ang A., Krousel-Wood M., Ward H. Predictive Validity of a Medication Adherence Measure in an Outpatient Setting. The Journal of Clinical Hypertension. 2008;10(5):348-354.

11. Vlaar P., Svilaas T., van der Horst I. et al. Cardiac death and reinfarction after 1 year in the Thrombus Aspiration during Percutaneous coronary intervention in Acute myocardial infarction Study (TAPAS): a 1-year follow-up study. The Lancet. 2008;371(9628):1915-1920.

12. Steg P., James S., Atar D. et al. ESC Guidelines for the management of acute myocardial infarction in patients presenting with ST-segment elevation. European Heart Journal. 2012;33(20):2569-2619.

13. O'Gara P., Kushner F., Ascheim D. et al. 2013 ACCF/AHA Guideline for the Management of ST-Elevation Myocardial Infarction. Circulation. 2013;127(4):e362-425.

14. Lagerqvist B., Fröbert 0., Olivecrona G.K. et al. Outcomes 1 Year after Thrombus Aspiration for Myocardial Infarction. New England Journal of Medicine. 2014;371(12): 1111-1120.
15. Jolly S.S., Cairns J.A., Yusuf S. et al. Randomized Trial of Primary PCI with or without Routine Manual Thrombectomy. New England Journal of Medicine. 2015;372(15):1389-1398.

16. Levine G.N., Bates E.R., Blankenship J.C. et al. 2015 ACC/AHA/SCAI Focused Update on Primary Percutaneous Coronary Intervention for Patients With ST-Elevation Myocardial Infarction: An Update of the 2011 ACCF/AHA/SCAI Guideline for Percutaneous Coronary Intervention and the 2013 ACCF/AHA Guideline for the Management of ST-Elevation Myocardial Infarction. Circulation. 2016;133(11), 1135-1147.

17. Fernández-Rodríguez D., Regueiro A., Brugaletta S. et al. Optimization in Stent Implantation by Manual Thrombus Aspiration in STSegment-Elevation Myocardial Infarction. Circulation. Cardiovascular Interventions. 2014;7(3):294-300.

18. Spaulding E.M., Marvel F.A., Lee M.A. et al. Corrie Health Digital Platform for Self-Management in Secondary Prevention After Acute Myocardial Infarction. Circulation: Cardiovascular Quality and Outcomes. 2019;12(5):e005509

19. Eindhoven D.C., Hilt A.D., Zwaan T.C. et al. Age and gender differences in medical adherence after myocardial infarction: Women do not receive optimal treatment - The Netherlands claims database. European Journal of Preventive Cardiology. 2017;25(2):181-189.

20. Brieger D., Chow C., Gullick J. et al. Improving patient adherence to secondary prevention medications 6 months after an acute coronary syndrome: Observational cohort study. Internal Medicine Journal. 2018;48(5):541-549.

\section{ORCID and contributionship:}

Nataliya V. Izhytska - 0000-0002-7089-5810 A,B,E,F

Dmytro I. Besh - 0000-0002-8982-7578 A,B,D,E,F

Olesya M. Besh - 0000-0003-3349-1291 C,D

Adrian Y. Fil - 0000-0003-3777-8783 E,F

\section{Conflicts of interest:}

Authors declare no conflict of interest.

\section{CORRESPONDING AUTHOR Nataliya V. Izhytska}

19b/45 Lysenytska str., 79032, Lviv, Ukraine

e-mail:nataizhytska@gmail.com

Received: 10.09 .2019

Accepted: 18.02.2020

\footnotetext{
A - Work concept and design, B - Data collection and analysis, C - Responsibility for statistical analysis, D-Writing the article, $\mathbf{E}$-Critical review, $\mathbf{F}$ - Final approval of the article
} 